\title{
A review on the synthesis, surface modification and drug delivery of nanoparticles
}

\author{
Md Dipu Ahmed ${ }^{1}$, Kazi M Maraz ${ }^{2}$, Shahirin Shahida ${ }^{2}$, Ruhania Tarannum ${ }^{3}$ and Ruhul A Khan 2,* \\ ${ }^{1}$ Department of Chemistry, University of Tennessee, Knoxville, Tennessee 37996, USA. \\ ${ }^{2}$ Institute of Radiation and Polymer Technology, Bangladesh Atomic Energy Commission, Savar, Dhaka, Bangladesh. \\ ${ }^{3}$ Department of Mechanical \& Nuclear Engineering, Kansas State University, Manhattan, KS 66506, USA.
}

Global Journal of Engineering and Technology Advances, 2021, 08(02), 032-045

Publication history: Received on 05 July 2021; revised on 06 August 2021; accepted on 09 August 2021

Article DOI: https://doi.org/10.30574/gjeta.2021.8.2.0114

\begin{abstract}
Over the past few years, the evolution of nanotechnology has extended into a wide range of applications. Nanotechnology has now become a multidisciplinary science that applies to electronics, materials science, biomedical engineering, microbiology, etc. Recently, nanotechnology is being used in biomedical and pharmaceutical science. Among them drug delivery is set to spread rapidly. Application of nanotechnology in health sector also created a potential impact such as in the fields of immunology, cardiology, endocrinology, ophthalmology, and oncology. Nanoparticles are unique because of their large surface area and it has the potential to change the properties of a bulk number of materials. The surface of nanoparticles can be modified with the help of various polymers, organic and inorganic substances according to the specific application and their use. Nanoparticles are also utilized as nano shells in drug delivery systems and cancer therapy. Nano shells can recognize the cancer cells when they are injected into the cancer area. The heat generated by the light absorbing nano shells due to the application of the near infrared light successfully kills tumour cells leaving the noncarcinogenic cells intact. In this review article, nanoparticles, the health implication of nanoparticles and their synthesis are discussed.
\end{abstract}

Keywords: Nanoparticle; Nanotechnology; Surface modifications; Drug delivery

\section{Introduction}

Nanostructures and nanotechnology are an extensive and multidisciplinary areas of research and development activity that is increasing strenuously around the world: Nowadays, nanotechnology research has gain enormous attention among the scientists based on the world assessment of current innovations. The word Nano is basically originated from a Latin word, which means dwarf. The ideal size range provided by nanotechnology refers to one thousand millionth of a particular unit, therefore nanometer is one thousand millionth of a meter (i.e., $1 \mathrm{~nm}=10^{-9} \mathrm{~m}$ ). According to the European Commission's recommendation, nanomaterial is defined as a natural, incidental, or manufactured material including particles, in a free state or accumulated or as agglomerated. There are $50 \%$ or more of the particles in the number size dissemination, in the range 1-100 $\mathrm{nm}$ there is one or more external dimensions [1-2]. The branch nanotechnology is the science that particularly deals with the processes that occur at the molecular level and of Nano length scale size multidisciplinary science also covers several applications in other disciplines such as biophysics, molecular biology, and bioengineering. Nanotechnology has created a potential impact in various fields like medicine including immunology, cardiology, endocrinology, ophthalmology, and oncology [3]. Engineered nanomaterials have received a fast-growing concern in recent years due to their unique physical and chemical properties. Those properties are expected to open new novel avenues of different technological, environmental, and biological applications of these nanomaterials in catalysts, semiconductors, sensors, drug carriers, and personal care products. Nanoscience and

\footnotetext{
${ }^{*}$ Corresponding author: Ruhul A Khan

Institute of Radiation and Polymer Technology, Bangladesh Atomic Energy Commission, Savar, Dhaka, Bangladesh. 
nanotechnology are considered to be a revolution anticipated to cause tremendous impacts on human life in the near future [4].

To employ nanoparticles in clinical applications, several researches have been conducted. The first generation of nanoparticles used for such applications are primarily based on liposomes and polymer-drug conjugates. Liposomes, which are spherical vesicles with a lipid bilayer membrane structure, can encapsulate both hydrophilic and hydrophobic agents, protecting the cargo during circulation in the body for example small molecule drugs, nucleotides, proteins, imaging agents or radionucleotides [5]. Liposomes and polymer-drug conjugates must provide the rudiments for the field of advanced drug delivery based on nanotechnology, but still there are several drawbacks are remaining. The first liposome-based therapeutic, liposome-encapsulated doxorubicin, for the treatment of HIV-related Kaposi's sarcoma, and for the treatment of ovarian cancer and multiple myeloma was sanctioned by the US Food and Drug Administration (FDA) in 1995 [5-7]. This Review focuses on nanoparticles surface modification, composition, and applications of nanoparticles.

\section{Background and History}

Human curiosity and imagination repeatedly encourage to develop and implementation of advanced technology and science. Nanoparticles and structures have been used in fourth century AD, by the Roman, that showed one of the most fascinating examples of nanotechnology in the ancient world.

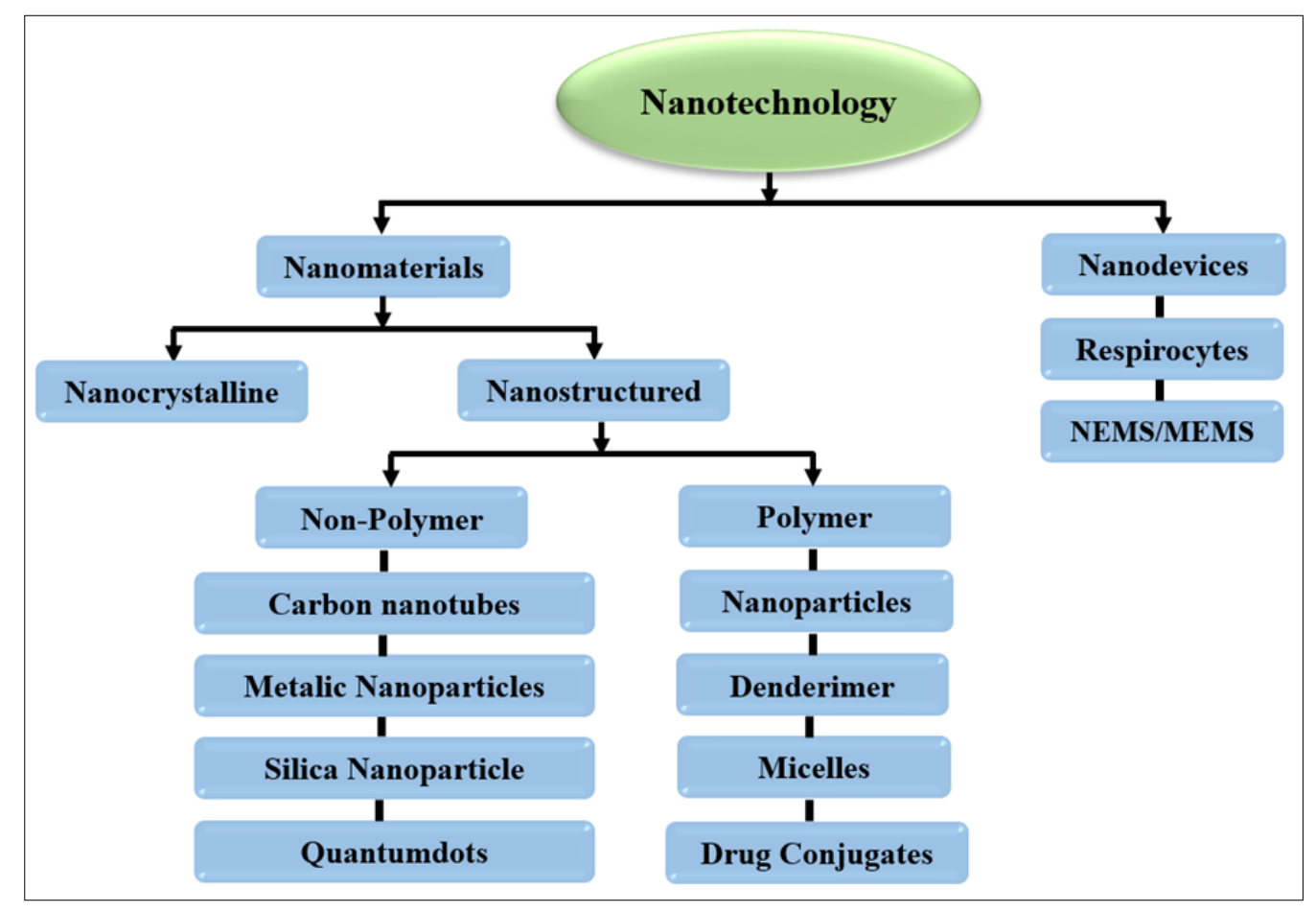

Figure 1 Classification and development of nanotechnology [14]

Nanoparticles were used by artisans as far back as Rome in the fourth century in the famous Lycurgus cup made of dichroic glass as well as the ninth century in Mesopotamia for creating a glittering effect on the surface of pots where nanoparticles were the secret of Glass Lycurgus Cup which had characteristic colour and Damascus steel which utilized to make swords. It is like just accidentally developed technique to manufacture Nano-objects and harness their innovative properties. However, after the development of the sophisticated device to analyse nanoscale materials, researchers are able to corroborate the development of nanotechnology, which has now become a subject of intense research [8-9]. Damascus steel was used to produce blades from about 500 A.D in Damascus. These blades are recognized for their resilience, sharpness, extreme strength and the aesthetic appeal of their unique surface pattern. Many researchers tried to reveal reason behind the special properties of Damascus steel and what they found was the presence of multi-walled carbon nanotube in the material. Glass Lycurgus Cup is the Roman period cup, i.e., fourth century AD. Modern analytic methods disclosed that Glass Lycurgus Cup comprises silver and gold nanoparticles in the ratio of roughly 7:3, with a size of $70 \mathrm{~nm}$ diameter. The cup demonstrates a unique colour display because of the presence of these metal nanoparticles. It appears green when observed in reflected light, for instance in daylight. 
However, it appears red when light is propagated through it [9]. It is now in the British museum. In modern times, pottery from the Middle Ages and Renaissance often retains a distinct gold- or copper-coloured metallic glitter. This luster is caused by a metallic film that was applied to the transparent surface of a glazing. The luster can still be visible if the film has resisted atmospheric oxidation and other weathering [10]. The luster originates within the film itself, which contains silver and copper nanoparticles. These nanoparticles are dispersed homogeneously in the glassy matrix of the ceramic glaze. well below a red heat, a remarkable change of properties takes place, whereby the continuity of the metallic film is destroyed. The result is that white light is now freely transmitted, reflection is correspondingly diminished, while the electrical resistivity is enormously increased.

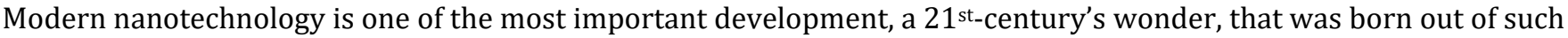
kind of imaginations. Even though human revelation to nanoparticles has appeared throughout human history, but it has significantly enhanced throughout the industrial revolution. Although nanotechnology is associated with modern science, they have a long history. The research of nanoparticles is not new thing. The conception of a "nanometer", it was first suggested by Richard Zsigmondy, who was Nobel Prize laureate in Chemistry back in the year 1925 . He invented the term nanometer specifically for characterizing particle size and was the first to measure the size of particles such as gold colloids using a microscope. Richard Feynman the 1965 Nobel Prize Laureate in physics, he is considered as the father of modern nanotechnology. He presented a lecture titled, "There's Plenty of Room at the Bottom" during the 1959 American Physical Society meeting at Caltech, in which he introduced the concept of manipulating matter at the atomic level. This novel idea demonstrated new ways of thinking and Feynman's hypotheses have since been proven correct [11-13]. Figure 1 represents the classifications of nanotechnology.

\section{Classifications and properties of nanoparticles}

Nanotechnology is one of the most propitious technologies of the 21st century that can convert the nanoscience theory to useful applications by observing, measuring, manipulating, assembling, controlling and manufacturing matter at the nanometer scale. Nanotechnology is defined as a combination of science, engineering, and technology that is conducted at the nanoscale ( 1 to $100 \mathrm{~nm}$ ), where the unique prodigies enable its applications in a different field, from chemistry, physics and biology, to medicine, engineering and electronic. This definition suggests the presence of two conditions for nanotechnology. The first is an issue of scale: nanotechnology is concerned to use structures by controlling their shape and size at nanometer scale. The second issue has to do with novelty: nanotechnology must deal with small things in a way that takes advantage of some properties because of the nanoscale $[12,14]$. The classifications and properties of nanoparticles are given below.

\subsection{Classifications of Nanoparticles}

Nanoparticles are generally classified based on their dimensionality, morphology, composition, uniformity, and agglomeration. Figure 2 represents the classifications of nanoparticles.

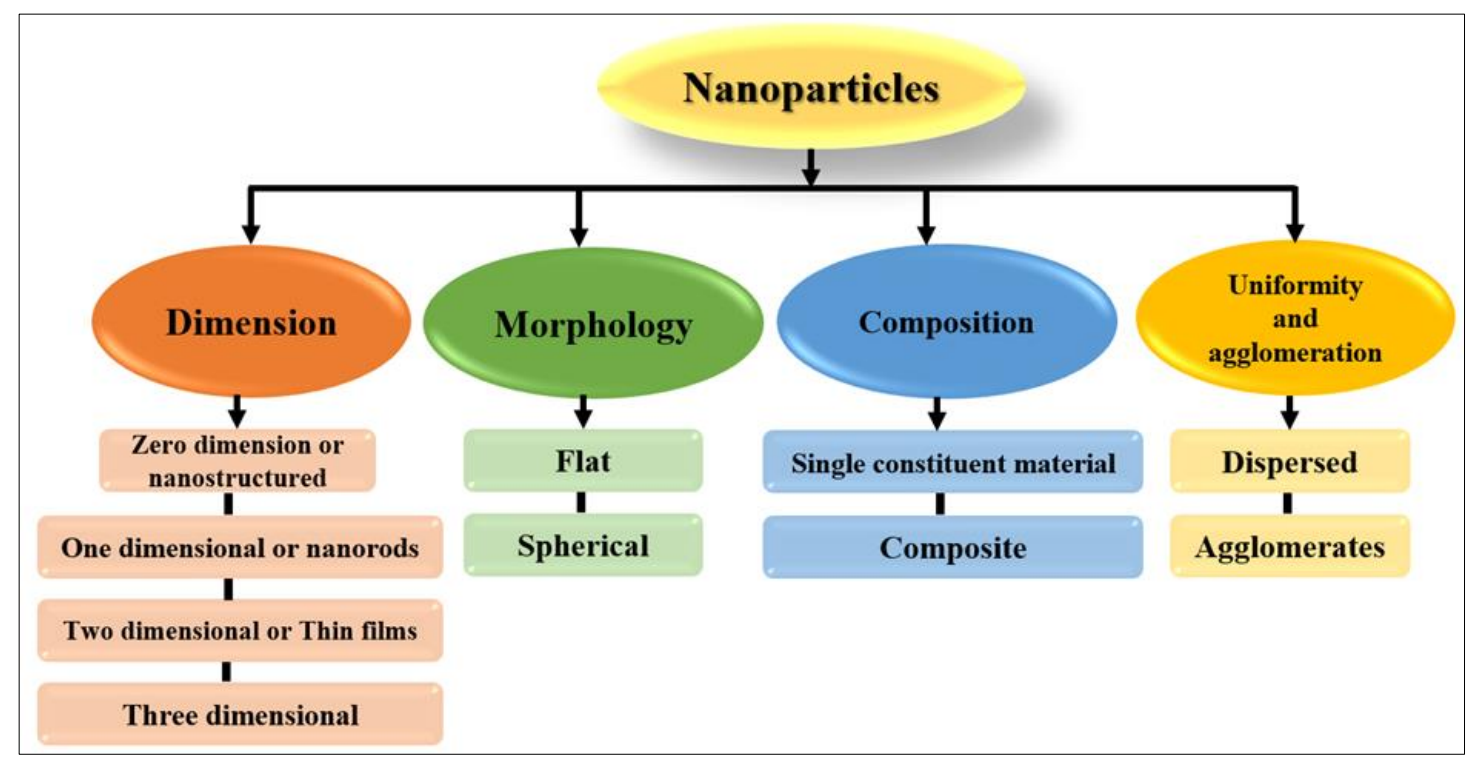

Figure 2 Classifications of Nanoparticle [16] 
In zero dimensional nanoparticles, the materials where in all the dimensions are measured within the nanoscale (no dimensions, or $0-\mathrm{D}$, are larger than $100 \mathrm{~nm}$ ). The most common representation of zero-dimensional nanomaterials are nanoparticles. For one dimensional nanoparticle, the dimension in the nanometer scale are typically thin films or surface coatings, and include the circuitry of computer chips and the antireflection and hard coatings on eyeglasses. These have been used in electronics, chemistry, and engineering. For examples, nanowires, nanorods, nanotubes, etc. Two dimensional nanoparticles, the nanomaterials have two dimensions in the nanometer scale. These include 2D nanostructured films, with nanostructures firmly attached to a substrate, or nanopore filters used for small particle separation and filtration. Asbestos fibres are an example of 2D nanoparticles. For example, nano-coatings, nano-films. Materials that are Nano-scaled in all three dimensions are considered 3D nanomaterials. These include thin films deposited under conditions that generate atomic-scale porosity, colloids, and free nanoparticles with various morphologies. For example, dendrimers, quantum dots, fullerenes (Carbon 60), (QDs) [15-16].

\subsection{Properties of Nanoparticles}

Nanoparticles have been explored in several biotechnological, pharmacological and pure technological applications. There is a link between bulk materials and atomic or molecular structures. Before to go forward, it is highly important to make some definitions for: Nanoscience, Nanotechnology, and Nanoparticles. Nanotechnology is the branch of technology that deals with dimensions and tolerances of less than 100 nanometers, especially the manipulation of individual atoms and molecules. Nanoscience scales, where the properties strongly differ in comparison to their largescale counterparts could be defined as the study of the phenomena and the manipulation of materials at atomic, molecular. A nanoparticle (or Nano powder or nanocluster or nanocrystal) is a microscopic particle with at least one dimension less than $100 \mathrm{~nm}$. The properties of nanoparticles are briefly discussed below.

\subsubsection{Physical Properties}

Nanoparticles are unique because of their large surface area and this special property dominates the contributions made by the small bulk of the material. Zinc oxide particles have been found to have superior UV blocking properties compared to its bulk substitute. This is one of the reasons why it is often used in the preparation of sunscreen lotions. Other examples of the physical properties of nanoparticles: Nanoparticles of yellow gold and Gray silicon are red in colour. Gold nanoparticles melt at much lower temperatures $\left(\sim 300{ }^{\circ} \mathrm{C}\right.$ for $2.5 \mathrm{~nm}$ size $)$ than the gold slabs $\left(1064{ }^{\circ} \mathrm{C}\right)$ [17].

\subsubsection{Optical Properties}

Nanoparticles also often exibit unexpected optical properties as they are small enough to confine their electrons and produce quantum effects. One example of this is that gold nanoparticles appear deep red to black in solution. The sizedependent optical property of them is due to change in the optical energy band gap, which in turn influences the surface plasmon resonance of the nanomaterials. The optical band gap increases with the decrease in particle size, especially for the semiconductor nanomaterials. Thus, the colloidal gold or silver nanoparticles produce different colours for different sizes, especially in the range of 1-10 $\mathrm{nm}$. Here it is pertinent to mention that surface plasmon resonance is the results of coherent excitation of the free electrons of the nanomaterials, which are present in the conductance band and in-phase resonance oscillations with the applied light energy. Hence the surface plasmon resonance is only observed if the particle size of the materials is less than the wavelength of the incident radiation [18].

\subsubsection{Magnetization Properties}

Other properties unique among nanoparticles are quantum confinement in semiconductor particles, surface plasmon resonance in some metal particles and super Para magnetism in magnetic materials. For example, ferroelectric materials smaller than $10 \mathrm{~nm}$ can switch their magnetization direction using room temperature thermal energy, thus making them unsuitable for memory storage. Thus, this property is not always desired in nanoparticles [19-20].

\subsubsection{Diffusion Properties}

At elevated temperatures especially, nanoparticles possess the property of diffusion. Sintering can take place at lower temperatures, over shorter time scales than for larger particles. Although this does not affect the density of the final product but there is a chance of agglomeration [21]. 


\section{Synthesis and Fabrication Methods of Nanoparticles}

synthetization and fabrication process of nanoparticles have been produced using several methods. These methods are primarily classified into bottom-up and top-down process shown in Figure 3. Top-down and bottom-up methods are two types of approaches used in nanofabrication.

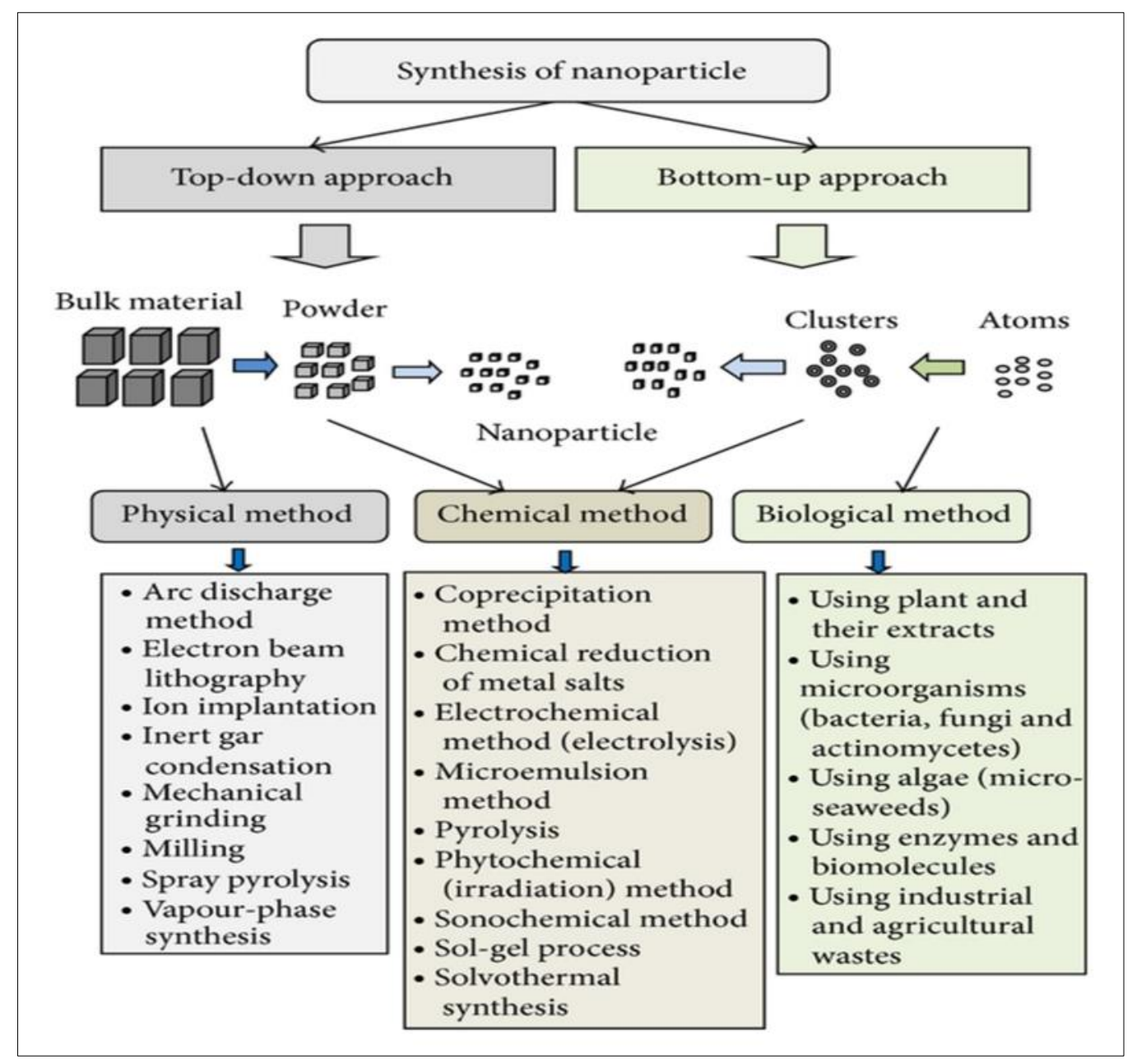

Figure 3 Synthesis and fabrication methods of nanoparticles [26]

In the bottom-up method, nanosized assemblies were formed by molecular components. A bottom-up synthesis method implies that the nanostructures are synthesized onto the substrate by stacking atoms onto each other, which gives rise to crystal planes, crystal planes further stack onto each other, resulting in the synthesis of the nanostructures. A bottomup approach can thus be viewed as a synthesis approach where the building blocks are added onto the substrate to form the nanostructures. Though the bottom-up approach often referred in nanotechnology, it is not a newer concept. All the living beings in nature observe growth by this approach only and also it has been in industrial use for over a century. In this technique, a nanometer structure is taken then using methods of assembly or self-assembly, a mechanism is developed which is larger than where it is started. Although the bottom-up approach is nothing new, it plays an important role in the fabrication and processing of Nano structures. Example: all cell uses enzymes to create DNA by taking constituent molecules and binding them together to make the final component.

On the other hand, a top-down synthesis method implies that the nanostructures are synthesized by etching out crystals planes (removing crystal planes) which are already present on the substrate. Attrition or Milling is a typical top-down method in making Nano particles, whereas the colloidal dispersion is a good example of bottom-up approach in the synthesis of Nano particles. However, the biggest problem with top-down approach is the imperfection of surface structure and significant crystallographic damage to the processed patterns. These imperfections which in turn leads to extra challenges in the device design and fabrication. But this approach leads to the bulk production of Nano material. Regardless of the defects produced by top-down approach, they will continue to play an important role in the synthesis 
of Nano structures. A top-down approach can thus be viewed as an approach where the building blocks are removed from the substrate to form the nanostructure.

The bottom-up approach is more advantageous than the top-down approach because the former has a better chance of producing nanostructures with less defects, more homogenous chemical composition, and better short- and long-range ordering compared to the top-down methods [2, 22-23]. Both approaches play very important role in modern industry and most likely in nanotechnology as well.

\subsection{Condensation in Inert Gas}

The inert gas condensation process is one of the most known and simplest technique for the fabrication of nanoparticles. A material, often a metal, is evaporated from a heated metallic source into a chamber which has been previously evacuated to about $10^{-7}$ torr and backfilled with inert gas to a low-pressure. The metal vapor cools through collisions with the inert gas atoms, becomes supersaturated and then nucleates homogeneously; the particle size is usually in the range 1-100 $\mathrm{nm}$ and can be controlled by varying the inert gas pressure. Ultimately, the particles are collected and may be compacted to produce a dense nanomaterial. The process utilizes a gas, which is typically inert, at pressures high enough to promote particle formation, but low enough to allow the production of spherical particles [24]. Metal is introduced onto a heated element and is rapidly melted. The metal is quickly taken to temperatures far above the melting point, but less than the boiling point, so that an adequate vapor pressure is achieved.

Gas is continuously introduced into the chamber and removed by the pumps, so the gas flow moves the evaporated metal away from the hot element. As the gas cools the metal vapor, nanometer-sized particles form. These particles are liquid since they are still too hot to be solid. The liquid particles collide and coalesce in a controlled environment so that the particles grow to specification, remaining spherical and with smooth surfaces. As the liquid particles are further cooled under control, they become solid and grow no longer [25]. At this point the nanoparticles are very reactive, so they are coated with a material that prevents further interaction with other particles (agglomeration) or with other materials.

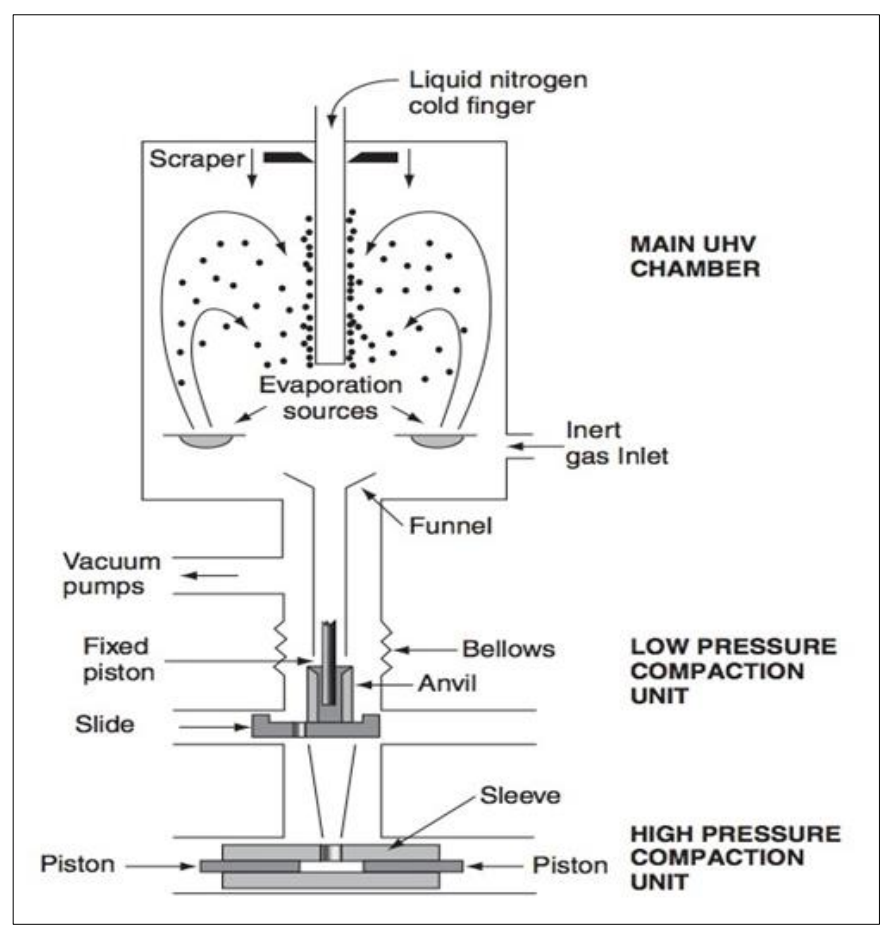

Figure 4 Nanoparticle condensation in inert gas [29]

\subsection{Chemical Vapor Deposition (CVD)}

Chemical vapor deposition (CVD) is one of the most common processes used to coat almost any metallic or ceramic compound, including elements, metals and their alloys and intermetallic compounds. There are several types of CVD process has been used, including atmospheric pressure chemical vapor deposition, metal-organic chemical vapor deposition, low pressure chemical vapor deposition, laser chemical vapor deposition, photochemical vapor deposition, 
chemical vapor infiltration, chemical beam epitaxy, plasma-assisted chemical vapor deposition and plasma-enhanced chemical vapor deposition. The CVD process involves depositing a solid material from a gaseous phase; this is achieved by means of a chemical reaction between volatile precursors and the surface of the materials to be coated. As the precursor gases pass over the surface of the heated substrate, the resulting chemical reaction forms a solid phase which is deposited onto the substrate. The substrate temperature is critical and can influence the occurrence of different reactions. Chemical vapor deposition (CVD) is a process in which films of materials are deposited from the vapor phase by the decomposition of chemicals on the surface of a substrate. Most frequently the process is thermally driven but photo- and plasma-assisted methods are also used.

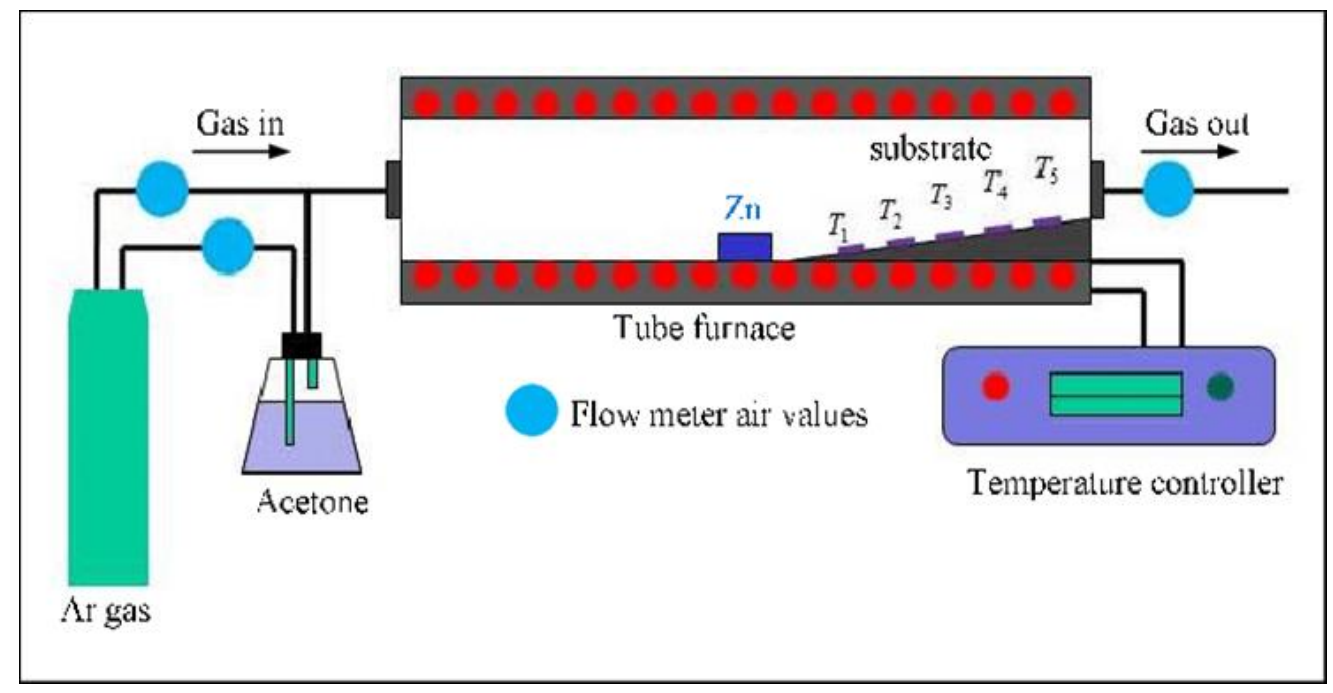

Figure 5 Chemical vapor deposition method [30]

The deposition of the film is controlled by a chemical reaction. The method is hence more versatile than many traditional methods. Growth is under non equilibrium conditions and the nature of the chemical precursor can in principle be used to control the phase deposited and its morphology. Other advantages of the method include a potential for both conformal and large-area growth, and the possibility of achieving, reproducibly, very high levels of purity in the asgrown materials [26-29].

\subsection{Atomic Layer Deposition (ALD)}

ALD is a method of applying thin films to various substrates with atomic scale precision. Similar in chemistry to CVD, except that the ALD reaction breaks the CVD reaction into two half-reactions, keeping the precursor materials separate during the reaction.

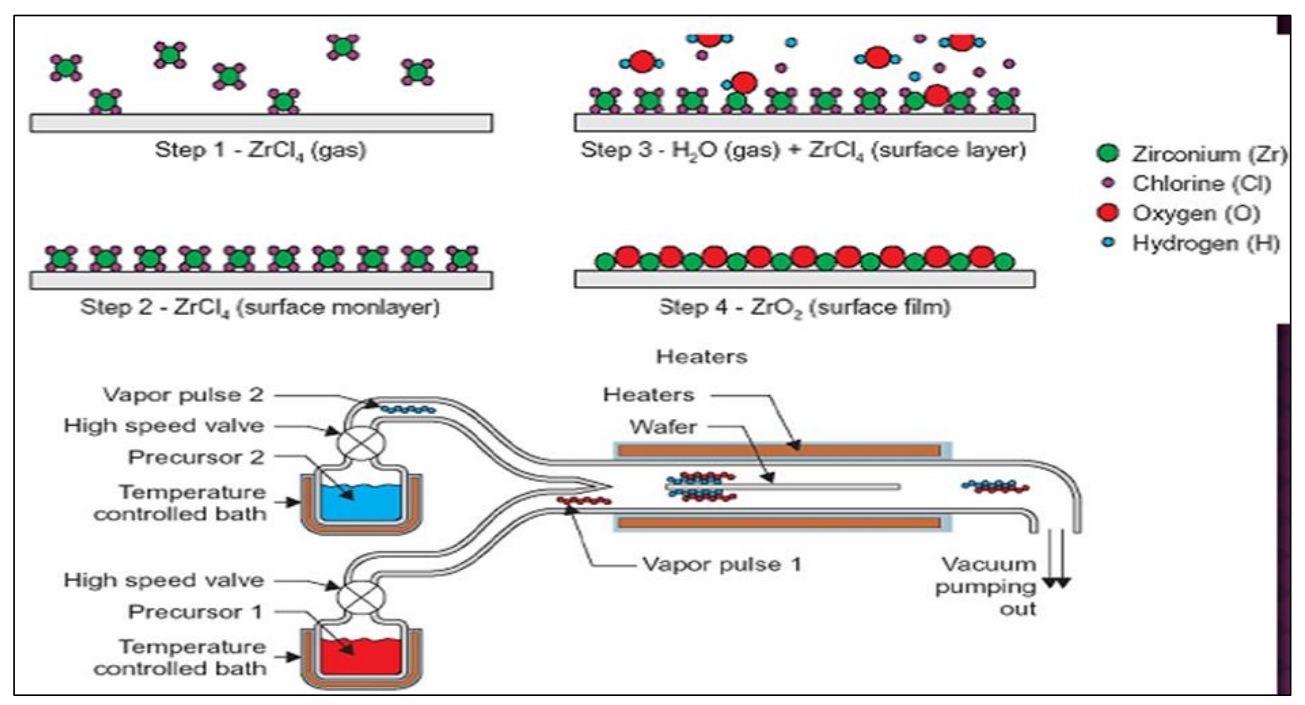

Figure 6 Atomic layer deposition method [35] 
ALD film growth is self-limited and based on surface reactions, which makes achieving atomic scale deposition control possible. By keeping the precursors separate throughout the coating process, atomic layer thickness control of film grown can be obtained as fine as atomic/molecular scale per monolayer.

This technique has been invented in 1977 by Dr. Tuomo Suntola in 1977, in the University of Helsinki in Finland. Dr. Suntola tried in fact to grow thin films of Zinc sulfide to fabricate electroluminescent flat panel displays. The main trick used for this technique is the use of a self-limiting chemical reaction to control in a very accurate way the thickness of the film deposited [30-33].

\subsection{Sol-gel Process}

The sol is a name of a colloidal solution made of solid particles few hundred nm in diameter, suspended in a liquid phase. The gel can be considered as a solid macromolecule immersed in a solvent. Sol-gel process consists in the chemical transformation of a liquid (the sol) into a gel state and with subsequent post-treatment and transition into solid oxide material. The main benefits of sol-gel processing are the high purity and uniform nanostructure achievable at low temperatures. Metal alkoxides and metal chlorides are typical precursors [34]. They undergo hydrolysis and polycondensation reactions to form a colloid, a system composed of nanoparticles dispersed in a solvent. The sol evolves then towards the formation of an inorganic continuous network containing a liquid phase (gel). Formation of a metal oxide involves connecting the metal centres with oxo (M-O-M) or hydroxo (M-OH-M) bridges, therefore generating metal-oxo or metal-hydroxo polymers in solution. After a drying process, the liquid phase is removed from the gel. Then, a thermal treatment (calcination) may be performed in order to favour further polycondensation and enhance mechanical properties.

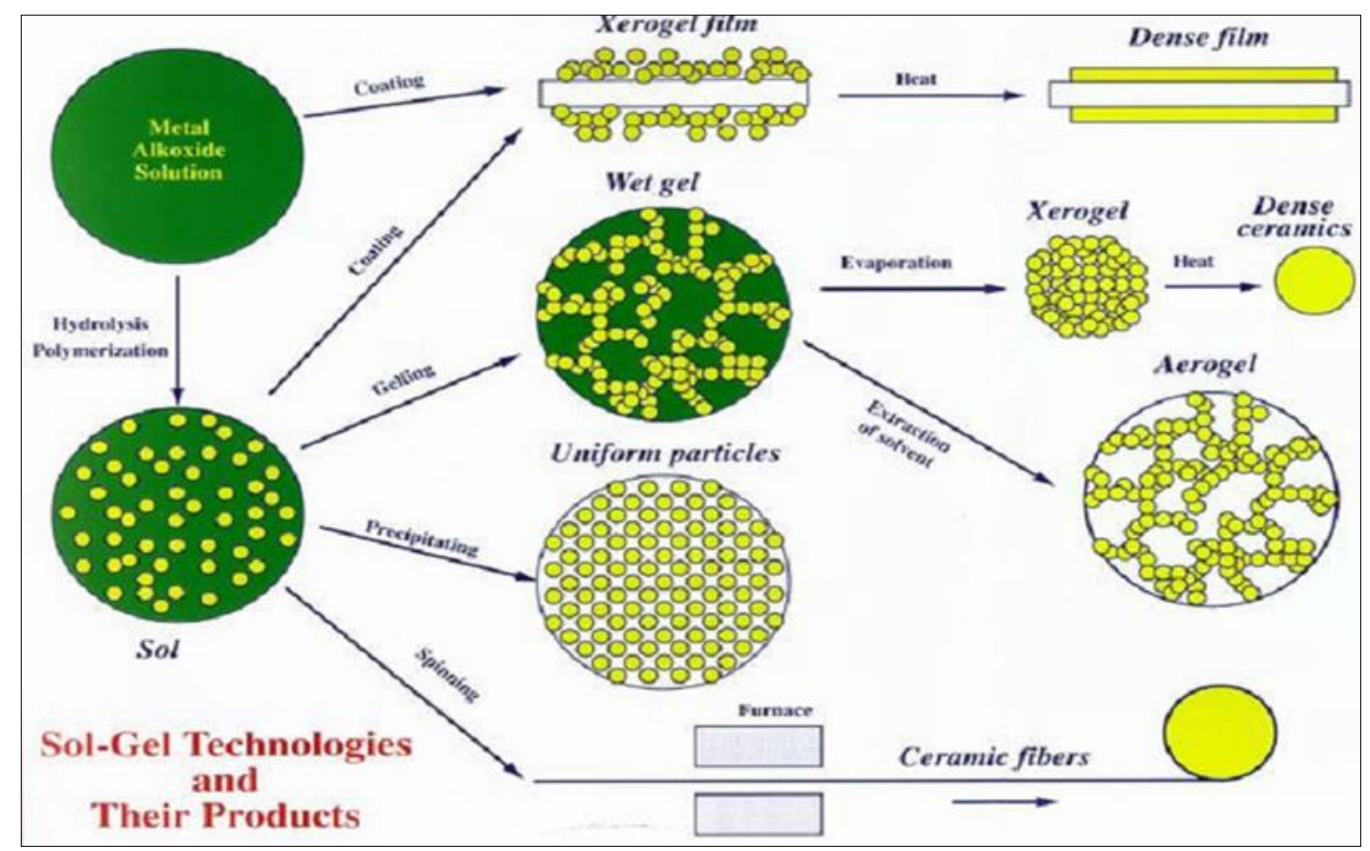

Figure 7 Sol gel method [41]

The sol-gel process is a wet-chemical technique used for the fabrication of both glassy and ceramic materials. In this process, the sol (or solution) evolves gradually towards the formation of a gel-like network containing both a liquid phase and a solid phase [30, 31, 35].

\section{Surface Modification and Drug Delivery: Drug Loading and Releasing}

Surface of nanoparticles can be modified by the help of various polymer, organic and inorganic substance according to the specific application and their use.

\subsection{Nanoparticles for Oral Delivery of Peptides and Proteins}

There is an urgent requirement of their suitable carrier system which still remains a challenge due to the fact that bioavailability of these molecules is limited by the epithelial barriers of the gastrointestinal tract and their susceptibility 
to gastrointestinal degradation by digestive enzymes. Polymer based nanoparticles facilitates the encapsulation of bioactive molecules and protect them against enzymatic and hydrolytic degradation. For example, nanoparticles have improved physicochemical properties of HIV drugs.

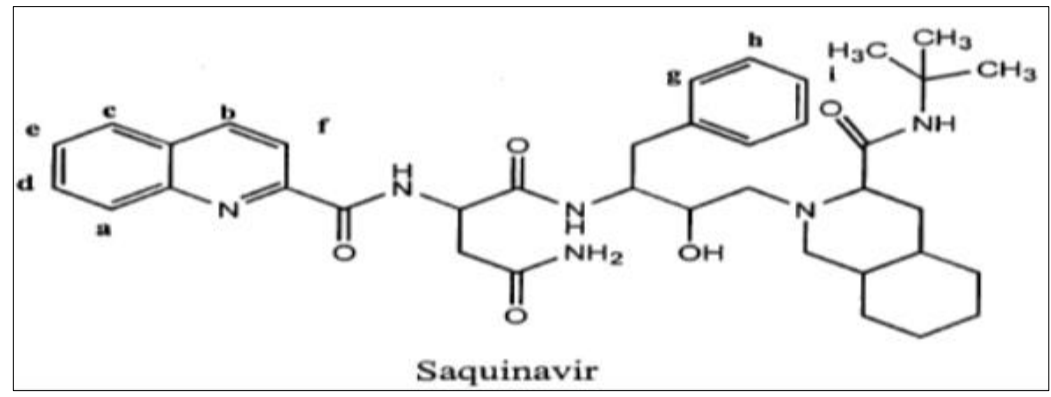

Figure 8 The Molecular structure of Saquinavir [43]

Effective oral administration of Saquinavir is a challenge. Saquinavir has poor solubility(35 $\mu \mathrm{g} / \mathrm{ml})$. Solubility can be increased 400-fold through encapsulation in cyclo-dextrinandpolyalkyl -cyanoacrylate nanoparticles(15.8mg/ml) [36]. The molecular structure of Saquinavir is given in Figure 8.

Nanoparticles also help to

- $\quad$ improve stability and solubility of drugs in Gastro Intestine (G.I.) tract

- Improve solubility of drugs in G.I tract

- Increase bioavailability of drugs

- $\quad$ Alter PK of drugs, e.g. extending half-life

- Targeting drugs to specific cells, tissues and organs

- Reduce toxicity of drugs

- Mitigate metabolism and drug-drug interactions (RIF and PI's)

\subsection{Tumour Targeting Using Nanoparticle Delivery Systems}

The rationale of using nanoparticles for tumor targeting is based on one of the most efficiency of nanoparticles is delivering drug in the area of the tumor targets because of enhancing permeability and retention effect. This can also be achieved by active targeting by ligands on the surface of nanoparticles [37]. Because of their small sizes, nanoparticles are taken by cells where large particles would be excluded or cleared from the body.

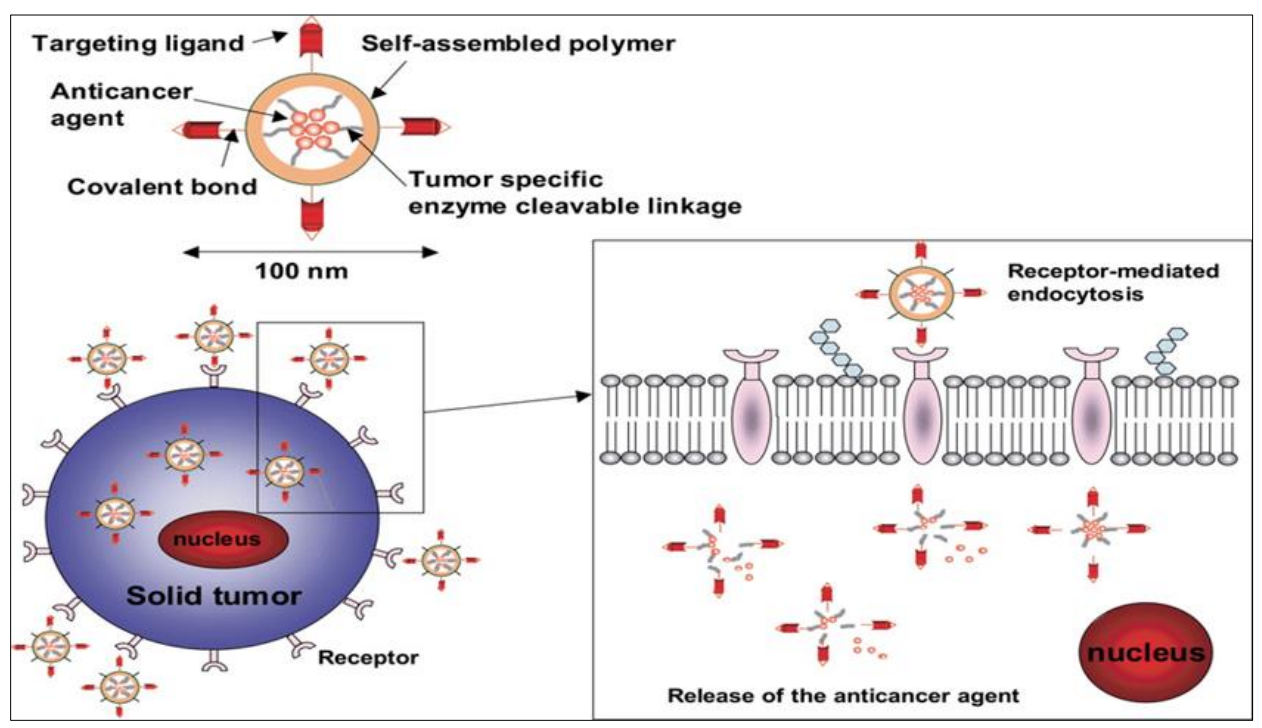

Figure 9 Nanoparticles for drug delivery in tumour treatment [46] 
A nanoparticle carries the pharmaceutical agent inside its core, while its shell is functionalized with a 'binding' agent. Through the 'binding' agent, the 'targeted' nanoparticle recognizes the target cell. The functionalized nanoparticle shell interacts with the cell membrane. The nanoparticle is ingested inside the cell, and interacts with the biomolecules inside the cell. The nanoparticle particles break, and the pharmaceutical agent is released [38]. The nanoparticles for drug delivery in tumour treatment is given in Figure 9.

\subsection{Nanoparticles for Drug Delivery into The Brain}

Nervous system is one of the most delicate microenvironments of the body which is protected by the blood-brain barrier (BBB) regulating its homeostasis. The blood- brain barrier (BBB) is the most important factor limiting the development of new drugs for the central nervous system. BBB is a highly complex structure that tightly regulates the movement of ions of a limited number of small molecules and of an even more restricted number of macromolecules from the blood to the brain, protecting it from injuries and diseases [39]. The BBB is characterized by relatively impermeable endothelial cells with tight junctions, enzymatic activity and active efflux transport systems. It effectively prevents the passage of water-soluble molecules from the blood circulation into the CNS, and can also reduce the brain concentration of lipid-soluble molecules by the function of enzymes or efflux pumps. BBB only permits selective transport of molecules that are essential for brain function, consequently, the BBB also significantly precludes the delivery of drugs to the brain, thus, preventing the therapy of a number of neurological disorders. As a consequence, several strategies are currently being sought after to enhance the delivery of drugs across the BBB. For example, polysorbate 80/LDL, transferrin receptor binding antibody (such as OX26), lactoferrin, cell penetrating peptides and melano transferrin have been shown capable of delivery of a self non transportable drug into the brain via the chimeric construct that can undergo receptor-mediated [40].

\subsection{Nano Shells as Cancer Therapy}

Nano-shells are the new modified forms of targeted cancer therapy, having core of silica and a metallic outer layer. The properties of nano-shells can be altered by simply tuning the core to shell ratio. This technology is being evaluated for cancer therapy. Nano-shells are tuned to absorb infrared rays when exposed from a source outside the body and get heated and cause destruction of the tissue. This has been studied in both in vitro and in vivo experiments on various cell lines Nano shells are injected into cancer area and they recognize cancer cells. Then by applying near-infrared light, the heat generated by the light-absorbing nano-shells has successfully killed tumour cells while leaving neighbouring cells intact. A model for the treatment of cancer using nanoparticles is shown in Figure 10.

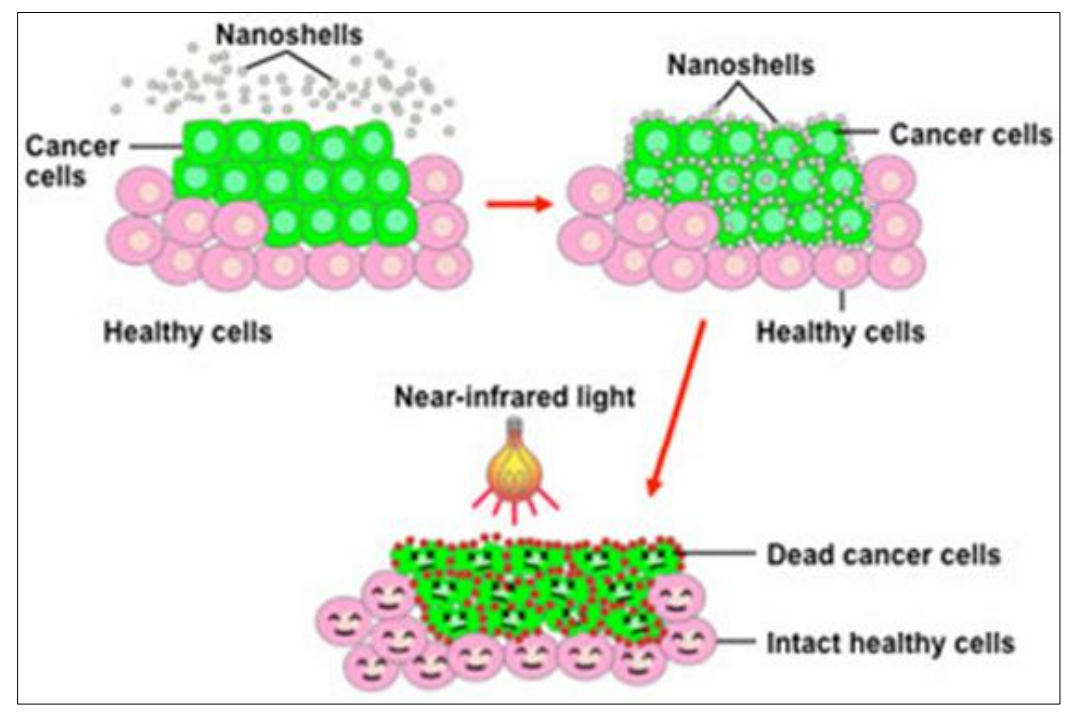

Figure 10 Treatment of cancer using nanoparticles [50]

Cancer nanotechnology, as a particular area of nanomedicine, is based upon the same premise that nanoparticles display unique properties potentially useful in medical (oncological) applications. Nanoparticles in the size range of 5-100nm have enough surface area to be properly functionalized to bind specific targets, with a variety of ulterior purposes [15, 41-42]. 


\section{Safety and waste Management}

Typically, nanomaterial safety (nano safety) in a research laboratory applies to engineered nano-sized materials only and does not apply to bulk micro-sized materials, bacteria, viruses, DNA, consumer products, etc. However, if NMs are treated or impregnated with the above-mentioned biological organisms, tissues or are used in laboratory animals, then both nano safety and biosafety procedures (or whichever is most stringent) will be implemented to protect the researchers and the environment.

\subsection{Precautionary Principle for Engineered Nanomaterials}

In the absence of complete scientific evidence, the potential threat of research materials on human health and the environment is assumed to be such that precautionary measures must be taken until the material is known to be safe. Lack of scientific certainty or cause and effect relationships should not be used as a reason for postponing reasonable measures that could prevent human exposure and environmental release. NMs can impart greater toxicity than microsized bulk materials of the same composition. NMs in insoluble form(s) can impart greater toxicity in the human body than micro-sized materials of the same chemical composition that are solubilized in water. NMs with fresh active surfaces are more reactive than encapsulated or passivated NMs (treated to reduce chemical reactivity of surface [43]. Unbound or free NPs can pose a more severe health hazard than the bound or embedded NMs. The duration, magnitude of exposure, and persistence of NMs in the human body can have adverse health effects.

\subsection{Standard Operating Procedures (SOPs)}

Researchers working with NMs should develop and implement written laboratory SOPs. The written SOPs should include environmental health and safety components such as PPE and respiratory protection, waste collection and disposal, and transportation of materials within the university and outside the university. Laboratory SOPs should be periodically reviewed and updated for effective control of potential hazards arising from NMs handling [44].

\subsection{Nanomaterial Specific Material Safety Data Sheet (MSDS)}

Each research laboratory should maintain a "Nanomaterial Inventory" and NM-specific MSDSs. MSDSs should be reviewed prior to handling the NMs in a research lab. If a NM-specific MSDS is not available, then the researcher should request one from the supplier or manufacturer. NM-specific MSDSs must include product description, hazard identification, composition, physicochemical characteristics, fire-fighting measures, accidental release measures, handling and storage, exposure controls/personal protection and disposal considerations [43, 45].

\subsection{Waste Management}

Though EPA has not promulgated NM specific regulations for waste disposal, many NMs may contain toxic components that are regulated. Therefore, every effort should be made by the researchers to prevent the release of NMs to the environment to the greatest extent feasible and comply with regulatory standards. Researchers must follow EHS protocols for management and disposal of chemical waste. NM-containing waste should be treated as hazardous waste. Laboratory generated NMs waste must not be disposed of in the regular trash or down the drain. NM-containing wastes including NM dispersions must be disposed of through EHS. NMs waste may be stored in a fume hood until ready for pickup, but must be stored in a closed container. Gloves, sharps and needles should be disposed of as bio-hazardous waste. NM contaminated coveralls should be disposed of as hazardous waste. Unused NM solids and NM dispersed solutions must be identified on the hazardous waste label [46].

\section{Conclusion}

Nanotechnology is a rapidly growing-area of research due to its wide range of application in different fields. The development of nanoparticles and nanotechnology in various fields-of has been extended in different directions, to observe things from micro to nano, to even smaller scale sizes by different microscopes in physics, from micro size bulk matter to small size carbon dots in chemistry, from room size computers to mobile slim size laptops in computer science, and to observe deeply the behavior of the cell's nucleus to study single complicated biomolecules at the nano level in biological science.

However, the most significant approaches in nanotechnology are clinical applications especially in drug delivery and cancer therapeutics. Recent developments of nanomaterials are made in the fields of physics, chemistry and material sciences have provided a number of nanomaterials with unique properties, which are expected to improve the treatment for many cases of tumors otherwise resistant to current therapies. This will be possible by merit of 
nanomaterials intrinsic cytotoxic activity and/or because of their capability to act as nanocarriers to deliver therapeutic molecules, such as drugs, proteins, nucleic acids or immune agents. These innovative biomedical applications are currently exploited in a variety of clinical trials and, in the near future, may support major development in the therapy of cancer.

\section{Compliance with ethical standards}

\section{Acknowledgments}

The research work was supported by the Annual Development Project (ADP) of Bangladesh. The title of the project is: "Strengthening of existing gamma source of Bangladesh Atomic Energy Commission". Special thanks to The Ministry of Science and Technology and The Ministry of Planning, Government of the People's Republic of Bangladesh.

\section{Disclosure of conflict of interest}

All authors state that there is no conflict of interest.

\section{References}

[1] N Raura, A Garg, A Arora, M Roma. Nanoparticle technology and its implications in endodontics: a review, Biomater. Res. 2020; 24(1): 1-8.

[2] AI Usman, AA Aziz, OA Noqta. Application of green synthesis of gold nanoparticles: A review, J. Teknol. Jan. 2019; 81(1): 171-182.

[3] S Thakkar, V Shah, MMR. patents on, and undefined 2017, Nanocrystal based drug delivery system: conventional and current scenario, ingentaconnect.com, Accessed: May 07, 2021.

[4] JJ Chieh, SM Mukhopadhyay, Y Cui. Editorial Multifunctional Nanomaterials for Biomedical Engineering: Unique Properties, Fabrications, and Diverse Applications, J. Nanomater. 2015.

[5] RA Petros, JM Desimone. Strategies in the design of nanoparticles for therapeutic applications, Nature Reviews Drug Discovery. Aug. 2010; 9(8): 615-627.

[6] Z Wenlong, F Gu, A Wang. Long-lasting eye drops using mucoadhesive nanoparticles View project Subsurface remediation and contaminated site characterization View project, Wiley Online Libr. May 2008; 83(5): 761-769.

[7] VP Torchilin. Targeted pharmaceutical nanocarriers for cancer therapy and imaging, AAPS Journal. 11 May 2007; 9(2).

[8] IC Ezema, PO Ogbobe, AD Omah. Initiatives and strategies for development of nanotechnology in nations: a lesson for Africa and other least developed countries, Nanoscale Res. Lett. Dec. 2014; 9(1): 1-8.

[9] S Sarina, E Waclawik, HZG. Chemistry, and undefined 2013, Photocatalysis on supported gold and silver nanoparticles under ultraviolet and visible light irradiation, pubs.rsc.org, Accessed: May 07, 2021.

[10] T Pradell, J Molera, C Bayés. P. R.-A. P. A, and undefined 2006, "Luster decoration of ceramics: mechanisms of metallic luster formation," Springer. May 2006; 83(2): 203-208.

[11] JE Hulla, SC Sahu, AW Hayes. Nanotechnology: History and future, Hum. Exp. Toxicol. 2015; 34(12): 1318-1321.

[12] S Bayda, M Adeel, T Tuccinardi, M Cordani, F Rizzolio. The history of nanoscience and nanotechnology: From chemical-physical applications to nanomedicine, Molecules. 2020; 25(1): 1-15.

[13] V Yadugiri. R. M.-C. S. (00113891), and undefined 2010, “'Plenty of room'-fifty years after the Feynman lecture., search.ebscohost.com, Accessed. 07 May 07.

[14] F Allhoff. On the Autonomy and Justification of Nanoethics, Springer.

[15] S Bhatia. Nanoparticles Types, Classification, Characterization, Fabrication Methods and Drug Delivery Applications," in Natural Polymer Drug Delivery Systems, Springer International Publishing. 2016; 33-93.

[16] Hett A. Nanotechnology: small matters, many unknown. 2004.

[17] V Vorovsky, A Kovalenko, A. K.-F., and undefined 2018, Preparation of zinc oxide nanopowders doped with manganese, which have ferromagnetic properties at room temperature. Accessed: 07 May 2021. 
[18] S Shen, T Liu. JG.-A. optics, and undefined 1998, “Optical phase-shift detection of surface plasmon resonance," osapublishing.org, Accessed: 07 May 2021.

[19] H Rahimi, A Ghasemi. R. M.-J. of M. and, and undefined 2017, Magnetic properties and magnetization reversal mechanism of Nd-Fe-B nanoparticles synthesized by a sol-gel method, Elsevier, Accessed: 07 May 2021.

[20] C An, M Alfi, B Yan. J. K.-J. of N. G. S. and, and undefined 2016, A new study of magnetic nanoparticle transport and quantifying magnetization analysis in fractured shale reservoir using numerical modeling, Elsevier, Accessed: 07 May 2021.

[21] O Habimana. K. S.-A. and, and undefined 2011, "Diffusion of nanoparticles in biofilms is altered by bacterial cell wall hydrophobicity," Am Soc Microbiol, Accessed: 07 May 2021.

[22] M Armbrüster, R Cardoso-Gil. Synthesis of Supported Ga Nanodrops by a Bottom-up Route Dedicated to Professor Yuri Grin on the Occasion of His 60th Birthday, Wiley Online Libr. Jul. 2015; 641(8-9): 1453-1458.

[23] S Makhsin, K Razak, A Aziz, R Nordin. Study on Controlled Size, Shape and Dispersity of Gold Nanoparticles (AuNPs) Synthesized Through Seeded-growth Technique for Labeling Immunoassay, 07 May 2021.

[24] RP Moraes, RA Hutchinson, TFL Mckenna. Small-Particle High-Solid-Content Bimodal Latexes: Highly Crosslinked Small Particles as Pseudo-Inert Nanofillers, Wiley Online Libr. Jan. 2013; 7(1): 36-53.

[25] D Wang, H Wang. Flotation of coarse coal particles in a three-phase gas-solid-liquid fluidised bed, Int. J. Oil, Gas Coal Technol. 2018; 17(2): 222-237.

[26] C Liu, Y Yang, R Zhang. X. R.-C. materials science, and undefined 2008, Kinetic Monte Carlo simulation of \{1 11$\}$ oriented SiC film with chemical vapor deposition, Elsevier, Accessed: 07 May 2021.

[27] K Nakaso, B Han, K Ahn. M. C.-J. of aerosol science, and undefined 2003, Synthesis of non-agglomerated nanoparticles by an electrospray assisted chemical vapor deposition (ES-CVD) method, Elsevier, Accessed: 07 May 2021.

[28] YM Manawi, A Samara, T Al-Ansari, MA Atieh. materials A Review of Carbon Nanomaterials' Synthesis via the Chemical Vapor Deposition (CVD) Method, mdpi.com 2018.

[29] L Hoyos-Palacio, D Castro. I. O.-T.-J. of M., and undefined 2019, “Compounds of carbon nanotubes decorated with silver nanoparticles via in-situ by chemical vapor deposition (CVD)," Elsevier, Accessed: May 07, 2021.

[30] Y Kavanagh, M Alam. D. C.-T. S. Films, and undefined 2004, The characteristics of thin film electroluminescent displays produced using sol-gel produced tantalum pentoxide and zinc sulfide, Elsevier, Accessed: 07 May 2021.

[31] Y Lydon Kavanagh BSA Sc, D Cameron. Thin Film Electroluminescent Displays produced using Sol-Gel Methods," 1604. Accessed: 07 May 2021.

[32] K Cao, J Cai, B Shan, R. C.-S. Bulletin, and undefined 2020, Surface functionalization on nanoparticles via atomic layer deposition, Elsevier, Accessed: 07 May 2021.

[33] JA McCormick, BL Cloutier, AW Weimer, SM George. Rotary reactor for atomic layer deposition on large quantities of nanoparticles, J. Vac. Sci. Technol. A Vacuum, Surfaces, Film. Jan. 2007; 25(1): 67-74.

[34] GR Lee, JA Crayston. Sol-gel processing of transition-metal alkoxides for electronics, Advanced Materials. 1993; 5(6): 434-442.

[35] Z Chen. G. Y.-C. S. Reviews, and undefined 2015, "The reactivity of the active metal oxo and hydroxo intermediates and their implications in oxidations," pubs.rsc.org, Accessed. 07 May 2021.

[36] T Vyas, A. Shahiwala, M. A.-I. journal of pharmaceutics, and undefined 2008, "Improved oral bioavailability and brain transport of Saquinavir upon administration in novel nanoemulsion formulations," Elsevier, Accessed. 07 May 2021.

[37] H GC. drug metabolism and undefined 2018, Novel Nanoparticles for Tumor Targeting Drug Delivery, ingentaconnect.com, Accessed. 07 May 2021.

[38] L Cuculis, et al. Surfactants Alter Nanoparticle - Model Cell Membrane Interactions. 2013.

[39] K Schoknecht, Y Shalev. Blood-brain barrier dysfunction in brain diseases: Clinical experience, Wiley Online Libr. 2012; 53(6): 7-13.

[40] H Li, Z Ming Qian. Transferrin/Transferrin Receptor-Mediated Drug Delivery, Med. Res. Rev. 2002; 22(3): 225250. 
[41] P Henshaw. H. M.-J. of the N. Cancer, and undefined 1943, "Influence of irradiation-killed cells on tumor growth," academic.oup.com, Accessed: 07 May 2021.

[42] J West. N. H.-C. opinion in Biotechnology, and undefined 2000, "Applications of nanotechnology to biotechnology: Commentary," Elsevier, Accessed: 07 May 2021.

[43] DO Warner, BS Randall Glidden, B McGlinch, BD Steven. Wald-man, "REVIEWS OF EDUCATIONAL MATERIAL NMS Clinical Manual of Anesthesia. Atlas of Uncommon Pain Syndromes.” Accessed: 07 May 2021.

[44] DM Powers. Laboratory Quality Control Requirements Should be Based on Risk Management Principles, researchgate.net.

[45] H Shin. M. B.-J. of K. S. of Hazard, and undefined 2013, "A Study on the Consciousness of Fire-fighting Officers for the Establishment of Safety Measures for wooden Cultural Assets," koreascience.or.kr, Accessed: 07 May 2021.

[46] W Sun. M. B.-W. Management, and undefined 2015, "Measurement of chemical leaching potential of sulfate from landfill disposed sulfate containing wastes,” Elsevier, Accessed: 07 May 2021. 